\title{
Extracellular matrix alterations and acute inflammation; developing in parallel during early induction of pulmonary fibrosis
}

\author{
Kristina Rydell-Törmänen ${ }^{1,2}$, Kristofer Andréasson ${ }^{1}$, Roger Hesselstrand ${ }^{1}$, Juha Risteli ${ }^{3}$, Dick Heinegård ${ }^{1}$, \\ Tore Saxne $^{1}$ and Gunilla Westergren-Thorsson ${ }^{2}$
}

Pulmonary fibrosis is a hallmark of several systemic diseases such as systemic sclerosis. Initiation and early development is not well characterized, as initiation usually is unnoticed in patients, yet fibrosis has been considered a late event, occurring after an inflammatory phase. By utilizing an animal model, the starting point can be defined and the initiation process and early development thoroughly investigated. To investigate these processes from a systemic perspective, we choose a systemic administration route, instead of the more commonly used local administration. The aim of this work was to study the initiation of pulmonary fibrosis in an animal model and to investigate early alterations in connective tissue, cell turnover and acute immune response in lung parenchyma. Animals were injected subcutaneously with bleomycin, three times a week (w) for 1-4w (controls received saline). Total collagen was histologically assessed by Picro Sirius Red and Masson's Trichrome, collagen production by antibodies directed against N-terminal of procollagens I and III, proliferation by labeling with proliferating cell nuclear antigen, apoptosis by TUNEL and innate immunity by detecting neutrophils and macrophages. Total collagen was significantly increased at 1, 2 and $4 \mathrm{w}$ compared with controls. Procollagen I, was increased at $1 \mathrm{w}$ and remained increased, whereas procollagen III-staining was increased at $2 \mathrm{w}$, compared with controls. Myofibroblasts were increased at all times as were proliferation, whereas apoptosis was increased from $2 \mathrm{w}$. Neutrophils peaked at $1 \mathrm{w}$ ( $2779 \pm 820$ cells $/ \mathrm{mm}^{2}$ ) and gradually decreased, whereas macrophages peaked at $2 \mathrm{w}\left(135 \pm 29\right.$ cells $\left./ \mathrm{mm}^{2}\right)$. Subcutaneously administered bleomycin induces rapid alterations in connective tissue and cell turnover, suggesting a plasticity of the connective tissue. A transient neutrophilia is detected and increased number of macrophages likely represents a clearance process of said neutrophils. The study suggests fibrosis initiation and acute inflammation to occur in parallel in this model.

Laboratory Investigation (2012) 92, 917-925; doi:10.1038/labinvest.2012.57; published online 2 April 2012

KEYWORDS: animal model; bleomycin; myofibroblasts; neutrophils; procollagen I and III

Pulmonary fibrosis is present in a wide range of different diseases and conditions, such as autoimmune disorders and infection, but may also occur without any known cause. Pulmonary fibrosis and/or inflammation are the key features of a large complex of conditions termed diffuse parenchymal lung diseases (DPLD), which affects the lung tissue between endothelial and epithelial basement membranes. ${ }^{1}$ Classification and definition of DPLD were published in 2002 by American Thoracic Society and European Respiratory Society, ${ }^{2}$ highlighting the diversity and similarities of different DPLDs. Importantly, however, as of today no effective treatment directed against any kind of pulmonary fibrosis is available. ${ }^{3}$
Histologically, several distinct patterns of pulmonary fibrosis can be detected and are associated with different DPLDs. A commonly occurring pattern is nonspecific interstitial pneumonia (NSIP), ${ }^{1,4}$ which is characterized by relatively homogenous primarily inflammatory and fibrotic alterations, visible as accumulation of connective tissue in the alveolar septa. This histological pattern is often found in association with collagen-vascular diseases, such as systemic sclerosis. Fibrosis has previously been considered the end result of chronic inflammation, yet accumulating evidence indicates that fibrosis and inflammation are two separate pathways. ${ }^{5}$

\footnotetext{
${ }^{1}$ Section of Rheumatology, Department of Clinical Science, Lund University, Lund, Sweden; ${ }^{2}$ Section of LungBiology, Department of Experimental Medical Science, Lund University, Lund, Sweden and ${ }^{3}$ Department of Clinical Chemistry, Institute of Diagnostics, University of Oulu, Oulu, Finland

Correspondence: Dr K Rydell-Törmänen, PhD, Section of Rheumatology/LungBiology, Department of Clinical Science/Department of Experimental Medical Science, Lund University, BMC D12, S-221 84 Lund, Sweden.

E-mail: Kristina.Rydell-Tormanen@med.lu.se

Received 29 August 2011; revised 30 January 2012; accepted 31 January 2012
} 
To develop therapeutics that can reverse fibrosis, a better understanding of the initiation and developmental processes is essential. ${ }^{6}$ Early alterations can efficiently be investigated in animal models, and an often used model is the administration of bleomycin to induce structural alterations. The most common administration route is intratracheal (IT) or intranasal (IN), however, intraperitoneal (IP), intravenous (IV) and subcutaneous (SC) administrations are also used. IT/IN administration, thus applying bleomycin directly into the airways, results in prominent epithelial damage, which leads to heterogeneous central pulmonary fibrosis/scarring. ${ }^{7}$ In this model, acute inflammation is present within the first week, (w) and fibrosis can be seen around day 14, with a maximal response around days $21-28$, and resolves thereafter. ${ }^{7,8}$ In contrast, SC administration, either by repeated injections or mini pumps is known to induce a more heterogeneous distal fibrosis, often located in the subpleural compartment, with vascular involvement. ${ }^{9}$ In this model acute inflammation peaks around $1-2 \mathrm{w}^{10,11}$ and skin fibrosis initiated by this route of administration are known to persist at least $6 \mathrm{w}$ after cessation of bleomycin. ${ }^{12}$ If anything, the lung is even more sensitive to bleomycin than skin, as demonstrated by the occurrence of lung alterations before alterations in skin, despite the skin being the site of injection. Thus, the IT/IN administration route may not be the best way to mimic the histological pattern of NSIP, and the ensuing diseases. A better alternative may be to deliver bleomycin SC, using repeated SC injections or mini pumps ${ }^{10}$ which induce a more NSIP-like pulmonary fibrosis. ${ }^{13}$ By this administration route bleomycin exerts the greatest effect on the vascular endothelium, which most likely mimics the clinical situation of NSIP. By administering injections over severalw, the initiation phase can be investigated, as the repeated exposures sustain a fibrotic response and preclude a potential resolution.

The specific aim of this study was to elucidate the initiation phase of pulmonary fibrosis, and explore the early alterations of the connective tissue, cell turnover and innate immune response in a model of pulmonary fibrosis induced by SC administered bleomycin.

\section{MATERIALS AND METHODS}

\section{Mice}

C57/Bl 6 mice were purchased from MoB A/S (Ry, Denmark) used and kept housed under barrier conditions, with food and water ad libitum. All protocols were approved by the local ethics committee (Malmö/Lund, Sweden; number M275-10).

\section{Bleomycin Administration}

We utilized a modified model of bleomycin-induced pulmonary fibrosis, ie, SC injections creating a systemic administration; see Figure 1 for illustrational overview.

Bleomycin $(0.1 \mathrm{ml} ; 500 \mu \mathrm{g} / \mathrm{ml}$, bleomycin sulphate, Bleomycin Baxter, Baxter Medical AC, Kista, Sweden) was administered using a 30-gauge needle, SC in the back of the animal, three times a w for 1, 2, 3 or $4 \mathrm{w}$. An additional group was

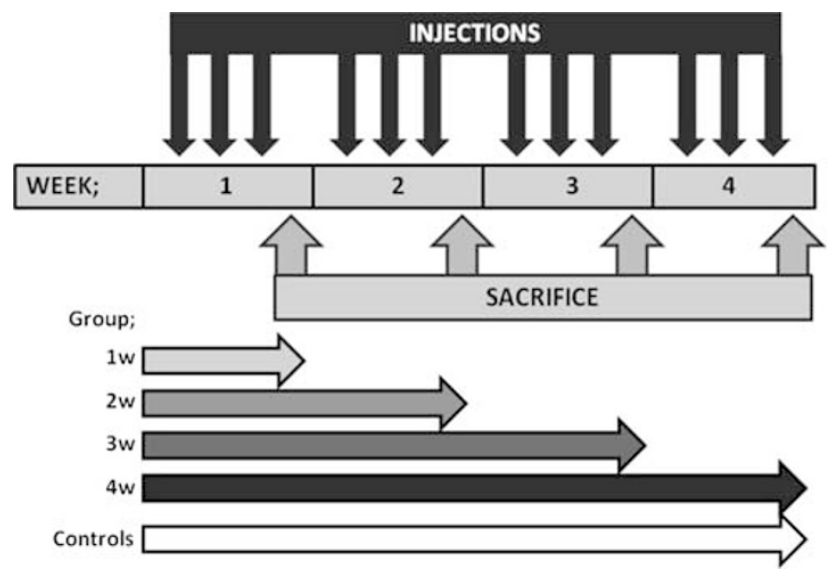

Figure 1 Schematic illustration of the study design. Animals were injected in the neck three times a week for $1-4 w$. Following killing the tissue specimens were obtained. Control animals received saline injections; animals subjected to different number of injections were compared but no differences were found between controls despite the number of injections.

killed following one single injection. Measures were taken to inject the bleomycin at a similar spot every time. Control animals received saline, preliminary experiments comparing histological parameter of animals subjected saline injections at various time points did not display any differences (data not shown), and therefore controls injected with saline for $4 \mathrm{w}$ were used in the study, $N=5-7$ in each group/time point. Animals were killed within $48 \mathrm{~h}$ of the last injection, when tissue samples were obtained for paraffin embedding.

\section{Tissue Processing and Routine Staining}

Tissue was fixed in $4 \%$ formaldehyde for $20-26 \mathrm{~h}$, dehydrated in $70 \%$ in several rinses over $2-3$ days before final dehydration and embedding in paraffin. Before analysis the paraffin embedded tissue were sectioned into $4-\mu \mathrm{m}$ slices and rehydrated from xylene in falling concentrations of ethanol to either Trisbuffered saline (TBS-buffer, used for immunohistochemistry) or tap water (total collagen staining and HTX/eosin). Sections were allowed to equilibrate for $15 \mathrm{~min}$ in buffer before continuous proceedings. To obtain an overall view of tissue and alterations, tissue was stained with HTX/eosin, according to standard protocol.

\section{Collagen Staining}

Two different methods were used to indentify collagen content in the tissue; Picro Sirius Red (PSR, visualizes fibrillar collagen as birefringent structures under polarized light) and Masson's Trichrome (MTC, labels connective tissue, primarily collagens, blue). Both staining methods were performed using standard protocols, dehydrated to xylene and mounted in permanent mounting medium. A total of 5-7 animals from each time point, including controls were investigated. 


\section{Picro Sirius Red}

$4-\mu \mathrm{m}$ thin sections were stained with Picro Sirius Red (PSR, Direct red 80, Alfa Eesar, Karlsruhe, Germany) $0.5 \mathrm{~g}$ dissolved in $500 \mathrm{ml}$ saturated picric acid. The slides were incubated for $1 \mathrm{~h}$, rinsed briefly in water and dehydrated in $99.9 \%$ ethanol.

\section{Masson's Trichrome}

$4-\mu \mathrm{m}$ thin sections were also used for staining with MTC (Masson's Trichrome Kit, Sigma-Aldrich, St Louis, MO, USA) according to manufacturers protocol, and dehydrated.

\section{Immunohistochemistry (IHC)}

Sections were processed for IHC according to a standard protocol; First sections were rehydrated and allowed to equilibrate in TBS-buffer for $15 \mathrm{~min}$. The primary antibody was applied, diluted in Dilution buffer (TBS supplemented with $1 \% \mathrm{BSA}$ ) and allowed to incubate (in a humid incubation chamber) in room temperature for $1.5-2 \mathrm{~h}$ or at $4{ }^{\circ} \mathrm{C}$ over night (both protocols produce equal results). The dilution of the primary antibody was determined by a dilution series based on the manufacturer's statement. To assess collagen synthesis antibodies directed against the N-terminal propeptides of procollagen type I (PINP) and type III (PIIINP) were used; to investigate number of myofibroblasts a double staining with PIIINP and $\alpha$-smooth muscle actin were utilized; to investigate proliferation an antibody directed against proliferating cell nuclear antigen (PCNA) was employed. The immune response was visualized using antibodies directed against macrophages and neutrophils. Following incubation with primary antibody, the slides were thoroughly rinsed in two changes of TBS buffer, before application of the secondary antibody and the nuclear marker DAPI. The dilution of the secondary antibody used was according to the manufacturer's recommendations (1:200) also diluted in dilution buffer. DAPI was used at a concentration of $30 \mu \mathrm{M}$ and further diluted 1:200 when mixed with the secondary antibody. The mixture of secondary antibody and DAPI were incubated for $45-60 \mathrm{~min}$ in room temperature, followed by rinsing as described above. The slides were mounted in fluorescent mounting medium and stored in $-20{ }^{\circ} \mathrm{C}$ before analysis. Before labeling macrophages, antigen-retrieval was needed; this was preformed enzymatically, using Proteinase K-solution $(20 \mu \mathrm{g} / \mathrm{ml})$ in phosphate buffered saline. The solution was applied onto the slide and incubated 15-20 min at room temperature. Apoptosis was detected using the TUNEL method according to the manufacturer's instructions (ApopTag Red In Situ, S7165, Millipore, Temecula, CA, USA). All details on the primary and secondary antibodies, in addition to required pretreatments, and suppliers can be found in Table 1. In all, 5-7 animals from each group (controls as well as bleomycin exposed animals) were included.

\section{Digital Imaging and Analysis}

Analysis was performed by digital imaging and the software ImageJ (v1.44j; Wayne Rasband, NIH, USA). All slides were analyzed in a blinded fashion. Images were taken of the lung parenchyma, as this area was considered to be the area of interest. The images were obtained in a random fashion, however, to assure the imaging of the regions of interest a starting point was selected-always the bottom part of the section close to (directly under) the lung parenchyma. From this starting point, the next image was taken 2-6 image fields'

Table 1 Summary of primary and secondary antibodies used in the study

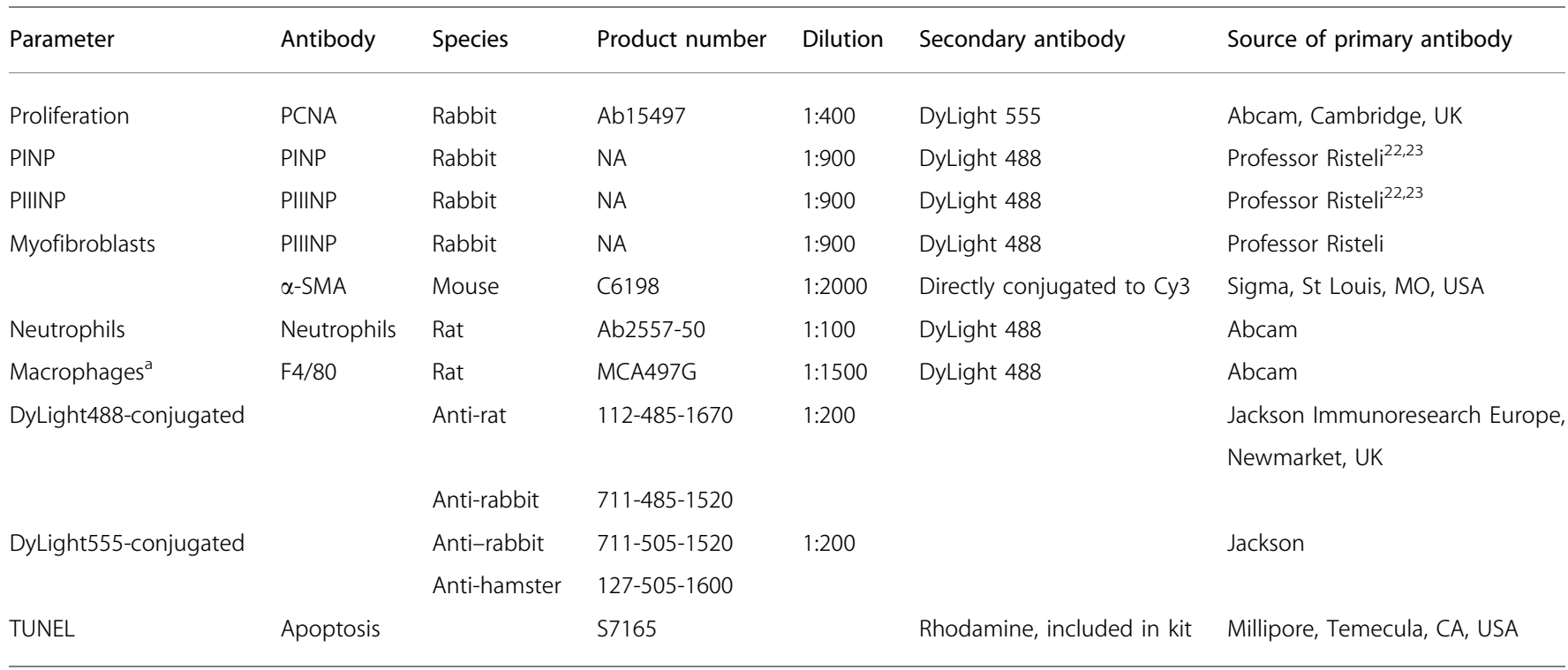

\footnotetext{
${ }^{\mathrm{a}}$ Macrophages required antigen retrieval, preformed as follows; proteinase $\mathrm{K}(20 \mu \mathrm{g} / \mathrm{ml}) 20 \mathrm{~min}$ in room temperature, before applying the primary antibody.
} 

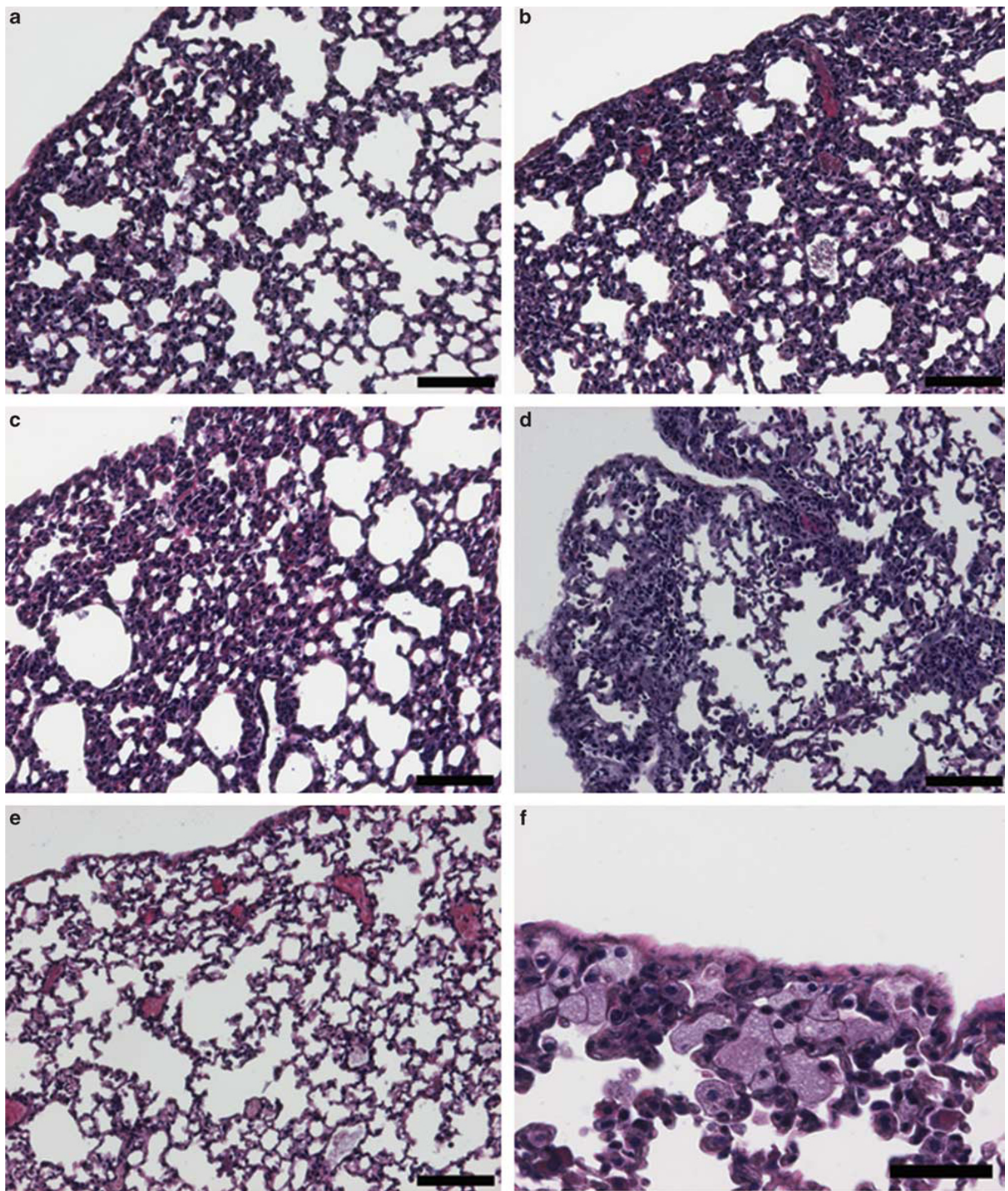

\section{$f$}

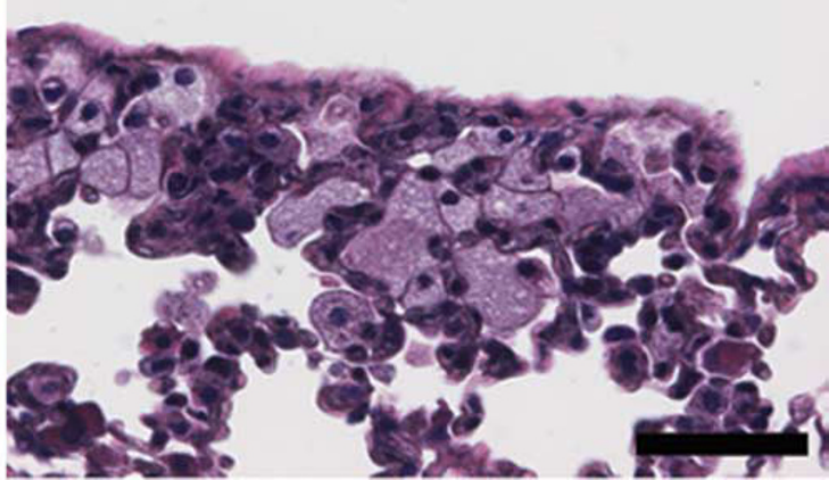

Figure 2 Representative images of lungs following repeated subcutaneous bleomycin injections. HTX staining of distal lungs from animals subjected to bleomycin exposure for $1 \mathrm{w}(\mathbf{a}), 2 \mathrm{w}(\mathbf{b}), 3 \mathrm{w}$ (c) and $4 \mathrm{w}$ (d) compared with a control (e). Cellular infiltrate were most prominent at 1 and $2 \mathrm{w}$, and remained only as patchy gatherings of cells at $4 \mathrm{w}$. At $4 \mathrm{w}$, numerous necrotic macrophages were found directly beneath the pleura (f). Scale bars represent $100 \mu \mathrm{m}$ in panels a-e and $50 \mu \mathrm{m}$ in panel $\mathbf{f}$. 

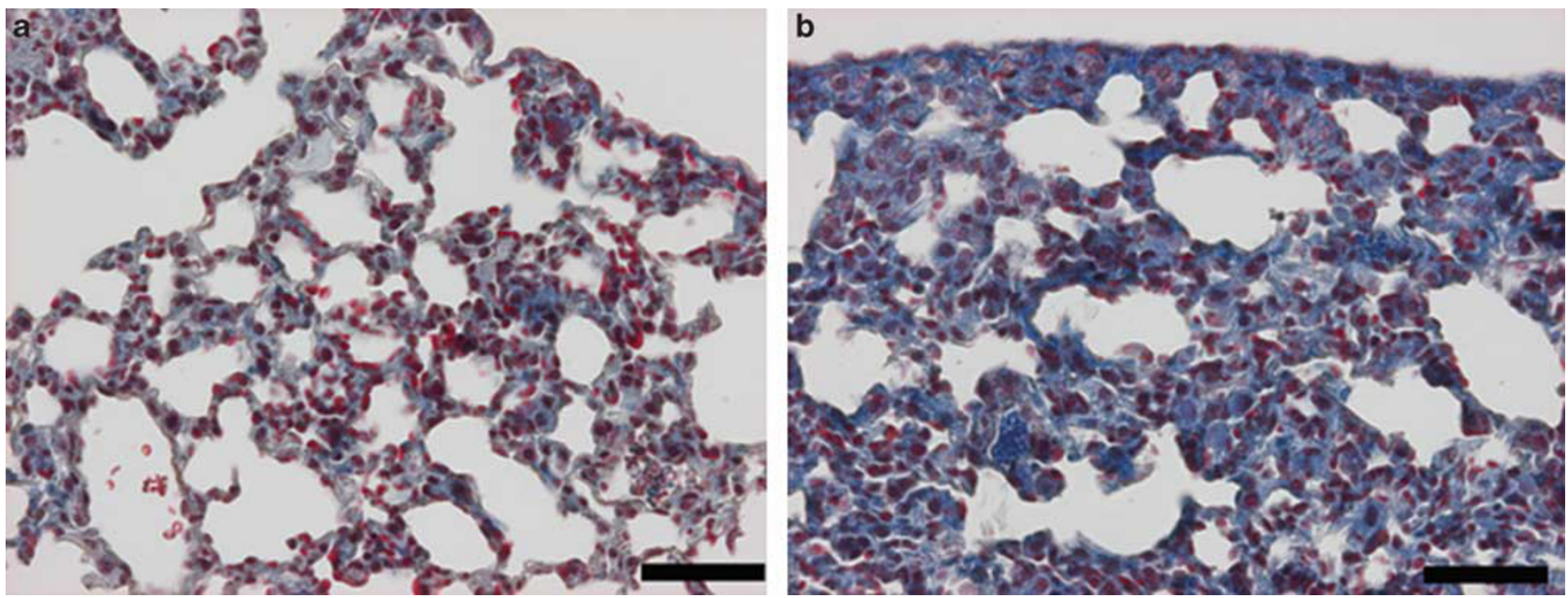

Figure 3 Images of lung parenchyma stained with Masson's Trichrome. The lung contains a relatively large amount of connective tissue, as can be seen in the control lung (a); however, this amount can be greatly increased following bleomycin exposure for 4w (b). Scale bar represents $50 \mu \mathrm{m}$.

right and 1-3 upwards from the starting image. A variable number of images of each slide were taken, ranging from 5 (when obtained at $\times 20$ magnification) to 10 (taken at $\times 40$ magnification). Specific analysis of the images was performed slightly differently depending on the parameter studied.

\section{Picro Sirius Red}

Slides were viewed under polarized light at $\times 40$ magnification and 8-10 randomly obtained images of the lung parenchyma were analyzed for area of positive staining $\left(\mu \mathrm{m}^{2}\right.$, red birefringence) and related to lung area $\left(\mathrm{mm}^{2}\right)$. The resulting data are given are positively stained area, per total tissue area $\left(\mu \mathrm{m}^{2} / \mathrm{mm}^{2}\right)$. However, to be comparable with other parameters of connective tissue (MTC, PINP and PIIINP) the final numbers were calculated as percentage labeled area of total area. Importantly, as the analysis was made using a dark background, the air spaces were not excluded in this analysis.

\section{Masson's Trichrome}

Large images of whole cross-sectioned lung were obtained at $\times 4$ (by taking several smaller images and stitching them together), and a region of interest (ROI) selected (lung parenchyma and distal lung were included, whereas central lung including larger airways and vessels were excluded). Within this ROI the total tissue area (excluding airspaces) was measured. The area of a defined nuance of blue corresponding to the intense blue collagen staining was measured and related to the total tissue. The results are given as proportion of collagen area of the tissue area (\%).

\section{Immunohistochemistry}

Depending on parameter, either the number of cells or the positively labeled area $\left(\mu \mathrm{m}^{2}\right)$ was calculated, and all results were related to $\mathrm{mm}^{2}$ of tissue area. Quantification was preformed as follows; the number of positively labeled cells was counted manually (except neutrophils), whereas the area and number of neutrophils was calculated digitally. The digital measurement of area was done by transforming the image to grayscale, and adjusting the threshold to incorporate only positively labeled area followed by a software calculation of the area. Neutrophils were counted digitally by a similar approach, as when measuring labeled area, instead the number of dots (ie, positively labeled cells) was counted by the software and related to area. To be comparable with other measurements of connective tissue, the results obtained for PINP and PIIINP were recalculated into fractions (\%) of labeled area in comparison with total tissue. Importantly, as the analysis was made using fluorescent microscopy, the air spaces were not excluded in this analysis.

\section{Statistical Analysis}

Results were statistically tested using Analyse-it for microsoft excel (Analyse-it Software, Leeds, UK). For determination of statistical differences between the different groups the nonparametric Kruskal-Wallis test combined with the LSD post hoc test was performed, and all time points were compared with controls. $P<0.05$ was considered statistically significant, and data are, if not stated otherwise, given as mean \pm s.d.

\section{RESULTS}

\section{Bleomycin Model}

All animals survived the experiment and no loss $>10 \%$ in body weight was detected. The relatively low dose of bleomycin did exert effects on the lung, first visualized in slides stained with HTX/eosin. At 1w a cellular influx was detected (Figure 2a) which peaked at $2 \mathrm{w}$ (Figure $2 \mathrm{~b}$ ). At $3 \mathrm{w}$ the cellular influx was resolving (Figure 2c) and at $4 \mathrm{w}$ (Figure 2d), only scattered aggregations of immune cells were found. Controls (Figure 2e), displayed a normal lung architecture compared with bleomycin-exposed mice. At $3 \mathrm{w}$ scattered macrophages 

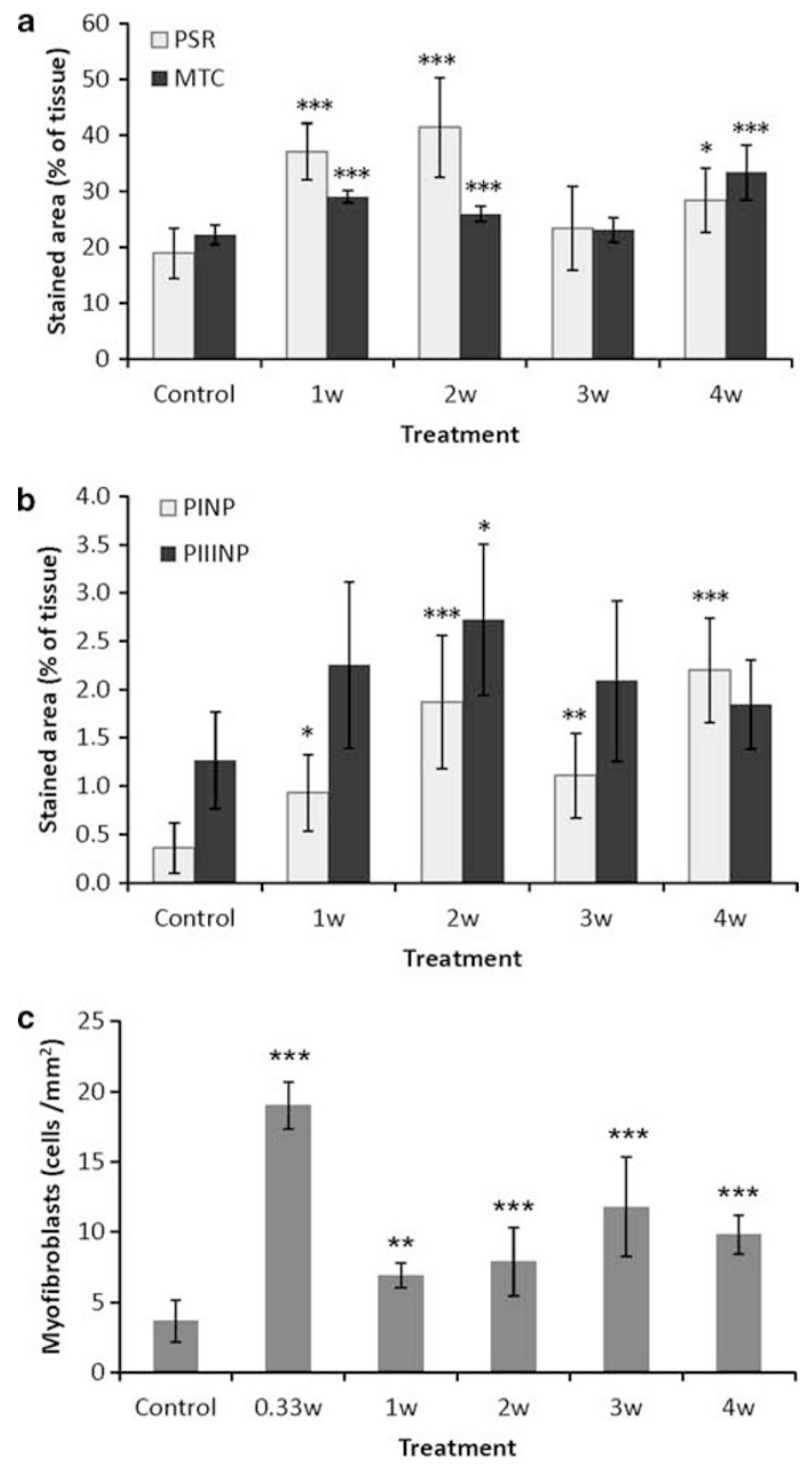

Figure 4 Alterations in connective tissue and myofibroblasts following bleomycin exposure. Variation of the two different parameters of total collagen (Picro Sirius Red (PSR) and Masson's Trichrome (MTC)) (a) and procollagen I and III (PINP and PIIINP) (b). Procollagens were detected using antibodies directed against the $\mathrm{N}$-terminal propeptides. ${ }^{26,27}$ All staining is calculated as area of total tissue area (\%). The number of myofibroblasts (defined as cells co-positive for PIIINP and $\alpha$-smooth muscle actin) was also altered because of bleomycin exposure (c), a significant increase present already at $1 \mathrm{w}$, which remained throughout the study. Myofibroblast data are given as mean \pm s.d. and ${ }^{*} P<0.05,{ }^{* *} P<0.01$ and ${ }^{* * *} P<0.001$ compared with controls. A total of 5-7 animals were analyzed for each parameter and time point.

appearing necrotic were found directly beneath the pleura and at $4 \mathrm{w}$ small assemblies of necrotic macrophages were found in the same localization (Figure 2f). Importantly, we did not find any evidence of neutrophil secondary necrosis, or the clearance system being overwhelmed. ${ }^{14}$

\section{Total Collagen Content and Collagen Synthesis}

Total collagen content was calculated using two separate methods (PSR and MTC), both displaying a similar patternincreases at 1 and $2 \mathrm{w}$, normalization at $3 \mathrm{w}$ and again increased levels at $4 \mathrm{w}$. MTC-stained sections (Figure 3) clearly showed relatively high amount of collagen present in normal lungs, but also the clear increase in apparent collagen content following 1, 2 and $4 \mathrm{w}$ of bleomycin exposure (4A). PSR-positive area was found to be significantly increased at 1,2 and $4 \mathrm{w}$ (Figure 4a). PINP was significantly increased at $1 \mathrm{w}$ and remained elevated throughout the study indicating elevated collagen synthesis (Figure $4 \mathrm{~b}$ ). In contrast, PIIINP was significantly increased only at $2 \mathrm{w}$, (although a tendency, $P=0.066$, was found at $1 \mathrm{w}$, Figure $4 \mathrm{~b}$ ). Interestingly, similar to PSR and MTC, PINP and PIIINP display a rapid increase at 1 and $2 \mathrm{w}$, at $3 \mathrm{w}$, respectively, however, all parameters but PINP (which does show a decrease, but remains significantly increased compared with controls) display a normalization.

\section{Increased Number of Myofibroblasts}

The number of myofibroblasts, here defined as cells co-positive for PIIINP and $\alpha$-smooth muscle actin, displayed a marked and early increase following bleomycin exposure (Figure 4c). The number of myofibroblasts rapidly increased compared with controls, already after one injection $(0.33 \mathrm{w})$, and this increase remained throughout the study. Interestingly, a difference in localization was noted at different time points; at $0.33 \mathrm{w}$ most positive cells were found in close proximity with vascular structures, whereas at later time points double-positive cells were found in the lung parenchyma as well.

\section{Cell Turnover-Proliferation and Apoptosis}

The number of proliferating cells (Figures $5 \mathrm{a}, \mathrm{c}, \mathrm{d}$ ) was significantly increased at all times. Proliferating cells were concluded to be mainly structural cells based on location. Double labeling with markers of smooth muscle and endothelial cells revealed no increased proliferation in these cell types (data not shown). The number of apoptotic cells (Figures $5 \mathrm{~b}, \mathrm{e}, \mathrm{f}$ ) was increased from $2 \mathrm{w}$ and onwards. Apoptosis in combination with the neutrophilia at 1 and $2 \mathrm{w}$, suggest a large proportion of apoptotic cells at this time to be neutrophils, whereas both structural and immunological cells appear to be apoptotic at 3 and $4 \mathrm{w}$.

\section{Immune Response}

The cellular immune response was found to be primarily consisting of neutrophils and macrophages (Figure 6). A prominent and rapid neutrophilia is present at $0.33,1$ and $2 \mathrm{w}$ and followed by a gradual decrease. The numbers, however, are still significantly increased compared with controls at $3 \mathrm{w}$. The number of macrophages displayed a significant increase at 1 and $2 \mathrm{w}$ parallel to neutrophils, but peaked $1 \mathrm{w}$ later, thus suggesting a clearance process. 

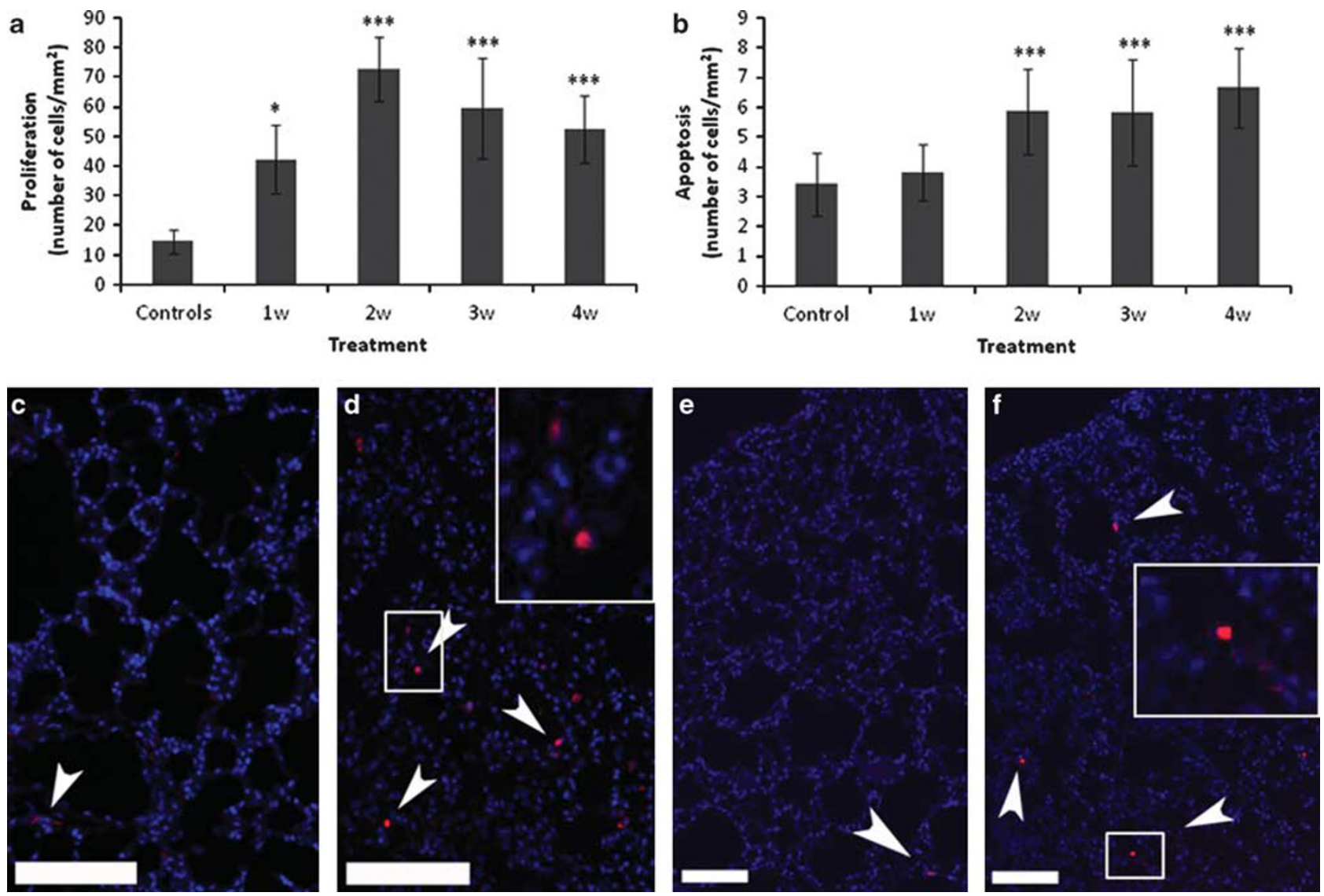

Figure 5 Cell turnover following bleomycin administration. The proliferation (a) was significantly increased at all times, whereas apoptosis (b) was increased from $2 \mathrm{w}$. Both methods resulted in red labeling of positive cells (arrowheads). To detect proliferating cells an antibody directed against proliferating cell nuclear antigen (PCNA) was used, detected by a DyLight 555-conjugated secondary antibody, and slides counterstained with DAPI. Proliferating cells was found in controls (c) but the number were increased at $2 \mathrm{w}$ (d). Apoptosis were visualized using a TUNEL-kit, detecting labeled cells by Rhodamine and also here the slides were counterstained with DAPI. Apoptotic cells were present in controls (e) and increased at $4 \mathrm{w}$ (f). Inserts show magnifications

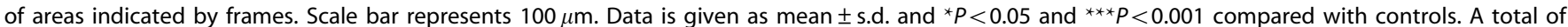
5-7 animals were analyzed for each parameter and time point.

\section{DISCUSSION}

The present study aimed to explore the initiation phase of pulmonary fibrosis, induced by continuous SC administrations of bleomycin, and investigate early changes in connective tissue, cell turnover and innate immune response. We found alterations of collagen and cell turnover to be rapidly occurring, thereby suggesting a greater plasticity of connective tissue than anticipated.

The early increase in collagen content of the lung parenchyma implies a rapid fibrotic response to bleomycin. Analogous to previously published reports of similar administration, the findings were located distally and found in the subpleural area. ${ }^{10,15}$ Early alterations are confirmed by studies investigating fibrosis initiated by SC insertion of an osmotic minipump filled with bleomycin. ${ }^{10,11}$

To investigate the synthesis of collagens I and III, immunohistochemistry was performed detecting the N-terminals of the propeptides of procollagen I and III. PINP displayed an early increase, indicative of upregulated synthesis, which was unexpected as collagen type I generally is considered to be accumulating in advanced stages. ${ }^{16}$ However, our data are in accordance with a recent study investigating IPF; procollagen I was found to be expressed in areas of early fibrosis. ${ }^{17}$ The results suggest that the increased total collagen is largely composed of collagen type I. The increases in PINP and PIIINP labeling should represent an increased synthesis of the respective procollagen, although in tissue the antibody labels both procollagen and the cleaved off propeptide. The latter is not much retained in tissues, where the level of free propeptide is very low (J Risteli, unpublished observation).

The number of myofibroblasts was increased at all times, even after one single injection, suggesting a plausible source of the increased collagen. Myofibroblasts have been implicated in the development of fibrosis and are known to synthesize connective tissue proteins rapidly. ${ }^{18}$ Interestingly, at $3 \mathrm{w}$ most connective tissue parameters normalized although not the number of myofibroblasts, which may suggest the normalization process to be due to increased degradation 

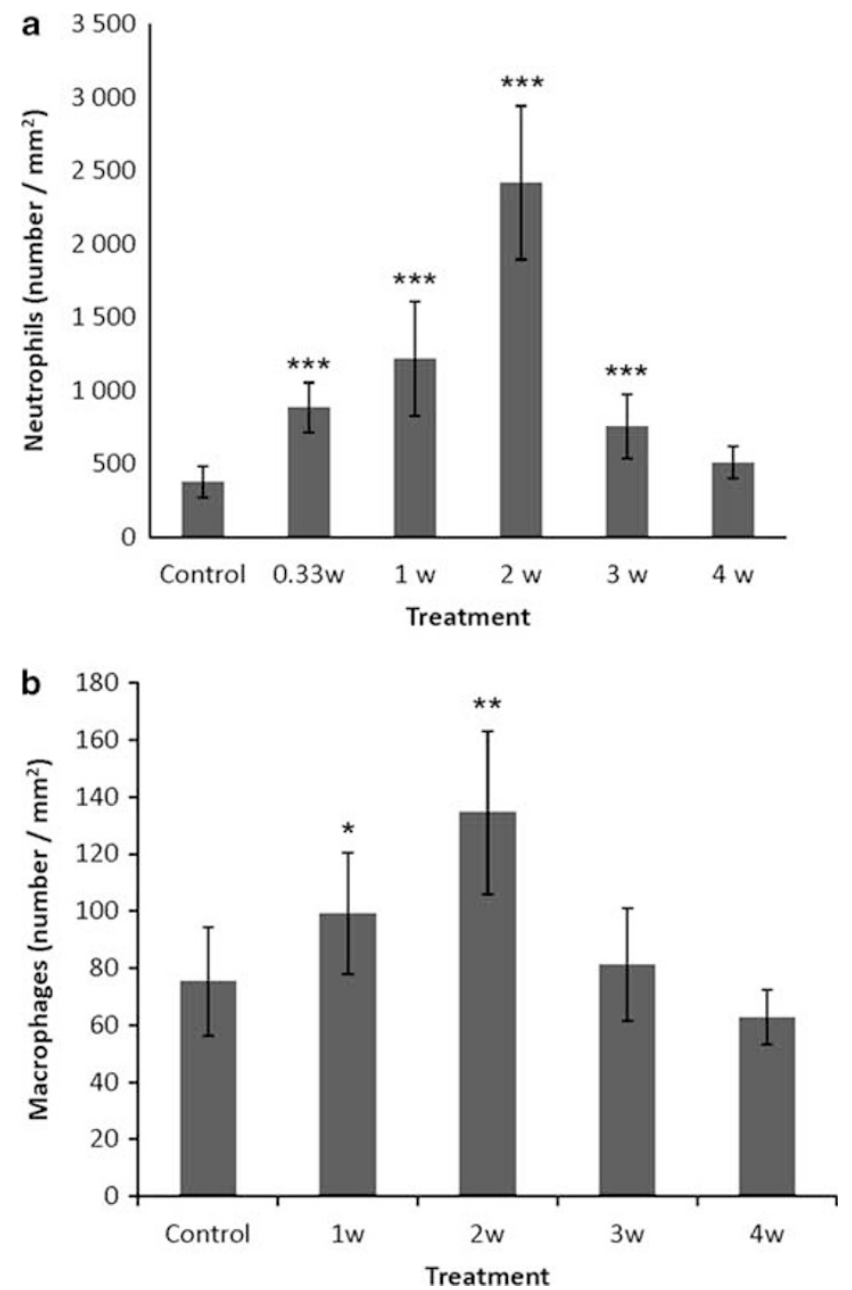

Figure 6 Innate immune response following repeated subcutaneous bleomycin injections. A transient neutrophilia was found to be statistically significant at 1 and $2 \mathrm{w}$ (a). At later time points no difference compared to controls was found. The number of macrophages was also increased at 1 and $2 \mathrm{w}(\mathbf{b})$, at a time when the neutrophilia was resolving. Data is given as mean \pm s.d. and ${ }^{*} P<0.05,{ }^{* *} P<0.01$ and ${ }^{* * *} P<0.001$ compared with controls. A total of 5-7 animals were analyzed for each parameter and time point.

rather than decreased synthesis. This finding will be further investigated.

An early proliferation of mesenchymal cells has been previously described by Aono et $a l,{ }^{10}$ at day 14, possibly explained by a proliferative response to a subtoxic dose of bleomycin. Bleomycin is described to induce tissue damage, ${ }^{15,19}$ thereby activating cellular repair mechanisms leading to fibrosis. The type of cells undergoing proliferation or apoptosis have not been thoroughly investigated; however, based on location (in alveolar tissue, in epithelium/endothelium and in the subepithelial/subendothelial area) and double-staining with markers for smooth muscle and endothelial cells, proliferating cells can be assumed to be of structural origin, most likely fibroblasts or fibroblast-like cells and a large proportion of the apoptotic cells identified throughout the study are neutrophils.
Neutrophilia is expected, as bleomycin is known to induce tissue damage and neutrophilia when administered IN/IT, ${ }^{7}$ and our results are in accordance with studies utilizing osmotic minipumps; ${ }^{10}$ a rapid increase within the first $\mathrm{w}$ and back to normal at $3 \mathrm{w}$. Studies using IT/IN administration does, however, describe a more rapid neutrophilia, most likely due to the direct administration of bleomycin to the airway epithelium. ${ }^{20}$ Macrophages were increased at the same time as the neutrophils started to decrease. It is known that macrophages clear away apoptotic neutrophils, and therefore the macrophages may represent a clearance mechanism. ${ }^{14}$ This is supported by the finding of macrophages appearing necrotic and full of engulfed material at 3 and $4 \mathrm{w}$. Interestingly, the innate immune response appeared to be resolved at $3 \mathrm{w}$, despite continuation of bleomycin injections.

The present study was, in contrast to many studies, analyzed primarily by histological techniques, allowing for determination of localization of alterations as well as quantification. The hydroxyproline method to determine collagen content in tissue is commonly used to quantify alterations in larger samples. It is, however, insensitive to local changes, and thus we concluded that it was unlikely that this technique would pick up subtle alterations in the lung parenchyma, considering the large amount of collagen normally present in the lung giving a high background level.

As this study was aimed at investigating the initiation phase of pulmonary fibrosis, we did not include analysis of the resolution phase. However, studies have shown skin fibrosis to persist for at least $6 \mathrm{w}$ after cessation of bleomycin, and the lungs are known to be more sensitive to bleomycin. ${ }^{12}$

Pulmonary fibrosis in man is known to be unresponsive to common therapeutics such as anti-inflammatory drugs, which can be explained by our findings; if inflammation occurs in parallel and not as a prequel to fibrosis, anti-inflammatory medication will have no effect on fibrotic alterations. A number of targets have been investigated as potentially anti-fibrotic in the bleomycin model; however, most of these have been administered at the same time or before bleomycin, thus acting preventive rather than therapeutic. ${ }^{21} \mathrm{~A}$ few substances have shown potential to actually resolve established fibrosis, such as a $5-\mathrm{HTR}_{2 \mathrm{~A} / \mathrm{B}}$ antagonist. ${ }^{22}$ To our knowledge none of these targets have been investigated neither in a SC model or proven effective in a clinical study.

The exact mechanism by which bleomycin induces fibrosis is not known, but is supposedly related to the cellular damage induced by the drug. ${ }^{23}$ Fibrosis is thought to be a result of cellular damage initiating an inflammatory response and activation of lung fibroblasts, and thus inhibition or modulation of proliferative signaling (such as TGF- $\beta$, HGF, tyrosine kinase, PDGF or $\mathrm{PGE}_{2},{ }^{9,10,24,25}$ which is in accordance with the increased proliferation and number of myofibroblasts presented in this paper. The specific signaling pathways of this model are still unknown, but most likely mimics the ones previously described. 
On the basis of our findings we conclude that bleomycin administered in a low dose via repeated SC injections, induce rapid and significant alterations of the extracellular matrix of the lung, detectable using histological techniques. The results implicates that the initiation of collagen production and fibrosis occurs in parallel to acute inflammation. Our findings furthermore suggest that low dose bleomycin administered SC is a more suitable model of NSIP than IT/IN administration, and highlight the need to take histological patterns of a disease into consideration when choosing a model.

This study presents new and intriguing aspects of the initiation and early development of pulmonary fibrosis, and may provide an explanation to why presently available treatments has no or very limited effect in the treatment of fibrotic diseases.

\section{ACKNOWLEDGEMENTS}

This work was made possible by grants from Royal Physiographic Society, Swedish Research Councel (Grant number: 11550), Swedish Heart-Lung Foundation, Kock's Foundation, Lars Hiertas Memorial Foundation, Österlund's Foundation, Gyllenstierna Krapperup's Foundation, The Sandberg Foundation and Faculty of Medicine, Lund University.

\section{DISCLOSURE/CONFLICT OF INTEREST}

The authors declare no conflict of interest.

1. Chilosi M, Murer B, Poletti V. Diffuse parenchymal lung diseases histopathologic patterns. In: Constabel U, du Bois RM, Egan JJ (eds). Prog Respir Res. Karger: Baselr, 2007, p. 44-57.

2. American Thoracic Society/European Respiratory Society International Multidisciplinary Consensus Classification of the Idiopathic Interstitial Pneumonias. This joint statement of the American Thoracic Society (ATS), and the European Respiratory Society (ERS) was adopted by the ATS board of directors, June 2001 and by the ERS Executive Committee, June 2001. Am J Respir Crit Care Med 2002;165:277-304.

3. Crestani B. The respiratory system in connective tissue disorders. Allergy 2005;60:715-734.

4. Martinez FJ. Idiopathic interstitial pneumonias: usual interstitial pneumonia versus nonspecific interstitial pneumonia. Proc Am Thorac Soc 2006;3:81-95.

5. Wynn TA. Cellular and molecular mechanisms of fibrosis. J Pathol 2008;214:199-210.

6. Veraldi KL, Hsu E, Feghali-Bostwick CA. Pathogenesis of pulmonary fibrosis in systemic sclerosis: lessons from interstitial lung disease. Curr Rheumatol Rep 2010:12:19-25.

7. Moore $\mathrm{BB}$, Hogaboam $\mathrm{CM}$. Murine models of pulmonary fibrosis. Am J Physiol Lung Cell Mol Physiol 2008;294:L152-L160.

8. Lawson WE, Polosukhin VV, Stathopoulos GT, et al. Increased and prolonged pulmonary fibrosis in surfactant protein C-deficient mice following intratracheal bleomycin. Am J Pathol 2005;167:1267-1277.
9. Yaekashiwa M, Nakayama S, Ohnuma $\mathrm{K}$, et al. Simultaneous or delayed administration of hepatocyte growth factor equally represses the fibrotic changes in murine lung injury induced by bleomycin. A morphologic study. Am J Respir Crit Care Med 1997;156: 1937-1944.

10. Aono $Y$, Nishioka $Y$, Inayama $M$, et al. Imatinib as a novel antifibrotic agent in bleomycin-induced pulmonary fibrosis in mice. Am J Respir Crit Care Med 2005;171:1279-1285.

11. Gupte VV, Ramasamy SK, Reddy R, et al. Overexpression of fibroblast growth factor-10 during both inflammatory and fibrotic phases attenuates bleomycin-induced pulmonary fibrosis in mice. Am J Respir Crit Care Med 2009;180:424-436.

12. Yamamoto T, Takagawa S, Katayama I, et al. Animal model of sclerotic skin. I: Local injections of bleomycin induce sclerotic skin mimicking scleroderma. J Invest Dermatol 1999;112:456-462.

13. Matute-Bello G, Frevert CW, Martin TR. Animal models of acute lung injury. Am J Physiol Lung Cell Mol Physiol 2008;295:L379-L399.

14. Rydell-Tormanen K, Uller L, Erjefalt JS. Direct evidence of secondary necrosis of neutrophils during intense lung inflammation. Eur Respir J 2006;28:268-274.

15. Fasske $E$, Morgenroth $K$. Experimental bleomycin lung in mice. A contribution to the pathogenesis of pulmonary fibrosis. Lung 1983; 161:133-146.

16. Gabrielli A, Avvedimento EV, Krieg T. Scleroderma. N Engl J Med 2009; 360:1989-2003.

17. Yamashita M, Yamauchi K, Chiba R, et al. The definition of fibrogenic processes in fibroblastic foci of idiopathic pulmonary fibrosis based on morphometric quantification of extracellular matrices. Hum Pathol 2009;40:1278-1287.

18. Baum J, Duffy HS. Fibroblasts and Myofibroblasts:What are we talking about? J Cardiovasc Pharmacol 2011:57:376-379.

19. Adamson IY. Pulmonary toxicity of bleomycin. Environ Health Perspect 1976;16:119-126.

20. Janick-Buckner D, Ranges GE, Hacker MP. Alteration of bronchoalveolar lavage cell populations following bleomycin treatment in mice. Toxicol Appl Pharmacol 1989;100:465-473.

21. Moeller A, Ask K, Warburton D, et al. The bleomycin animal model: a useful tool to investigate treatment options for idiopathic pulmonary fibrosis? Int J Biochem Cell Biol 2008;40:362-382.

22. Konigshoff $M$, Dumitrascu $R$, Udalov $S$, et al. Increased expression of 5hydroxytryptamine2A/B receptors in idiopathic pulmonary fibrosis: a rationale for therapeutic intervention. Thorax 2010;65:949-955.

23. Chen J, Stubbe J. Bleomycins: towards better therapeutics. Nat Rev Cancer 2005;5:102-112.

24. Dackor RT, Cheng J, Voltz JW, et al. Prostaglandin E2 Protects Murine Lungs from Bleomycin-Induced Pulmonary Fibrosis and Lung Dysfunction. Am J Physiol Lung Cell Mol Physiol 2011;301:L645-L655.

25. Mi S, Li Z, Yang HZ, et al. Blocking IL-17A Promotes the Resolution of Pulmonary Inflammation and Fibrosis Via TGF-\{beta\}1-Dependent and -Independent Mechanisms. J Immunol 2011;187:3003-3014.

26. Kaarteenaho-Wiik R, Sademies O, Paakko $P$, et al. Extracellular matrix proteins and myofibroblasts in granulomas of sarcoidosis, atypical mycobacteriosis, and tuberculosis of the lung. Hum Pathol 2007, 38:147-153.

27. Tormanen KR, Uller $\mathrm{L}$, Persson CG, et al. Allergen exposure of mouse airways evokes remodeling of both bronchi and large pulmonary vessels. Am J Respir Crit Care Med 2005;171:19-25. 